\title{
Serotonin-Deficient Mutants and Male Mating Behavior in the Nematode Caenorhabditis elegans
}

\author{
Curtis M. Loer and Cynthia J. Kenyon \\ Department of Biochemistry and Biophysics, University of California, San Francisco, California 94143
}

\begin{abstract}
Defining a behavior that requires the function of specific neurons in the free-living nematode Caenorhabditis elegans can allow one to screen for mutations that disrupt the specification or function of those neurons. We identified serotonin-immunoreactive neurons required for tail curling or "turning" behavior exhibited by $c$. elegans males during mating. Males mutant in three different genes that reduce serotonin expression, cat-1, cat-4, and bas-1, exhibited defects in turning behavior similar to those of wild-type males in which these neurons were ablated. The turning defect of cat -4 males was rescued by exogenous serotonin, consistent with the idea that their behavioral defect is caused by a lack of serotonin. While the serotonin-deficient mutants we analyzed shared certain behavioral traits, they were blocked for serotonin synthesis at different steps. Analysis of these and additional serotonin-deficient mutants may help us understand how a neuron controls the expression of a serotonergic phenotype.
\end{abstract}

[Key words: serotonin, biogenic amines, dopamine, neurotransmitter]

A neuron's choice of neurotransmitter is one critical decision required for the generation of a functional nervous system. One approach to asking how this decision is made is genetic: to isolate and analyze mutants that no longer make the neurotransmitter. Some mutations will be found to affect genes involved in terminal differentiation, for example, those encoding biosynthetic enzymes for the neurotransmitter; other mutations may alter genes encoding transcriptional regulators that coordinate the expression of the synthetic enzymes and other terminal differentiation genes. Thus, screening for mutants lacking expression

\footnotetext{
Received Dec. 21, 1992; revised June 3, 1993; accepted June 15, 1993.

Special thanks to Steve Salser, who coded hundreds of mating assay plates. Thanks are also due to many other members of the Kenyon lab for discussions, suggestions, strains, and coding of mating assays. Thanks to David Bentley, Bob Horvitz, and Gian Garriga for their comments on the manuscript. Thanks to Gian Garriga for providing the strain containing the mutation bas-I(ad446) and for suggestions regarding individual worm anti-serotonin staining. Thanks to Cori Bargmann for strains and suggestions. We thank the following persons for sharing their initial observations that led us to do these experiments and for other unpublished information that aided us: G. Garriga, M. Finney, C. Desai, S. McIntire, H. R. Horvitz, J. Lewis, A. Ruiz-Morales, J. Mancillas, J. Sulston, K. Liu, and P. Sternberg. Some of the nematode strains used were obtained from the Caenorhabditis Genetics Center, which is funded by the NIH National Centers for Research Resources. C.M.L. was supported by an NIH postdoctoral fellowship and traineeship. Funding for this work also came from a Searle Scholarship and a Packard Foundation fellowship to C.J.K. Thanks also to James McKerrow, Department of Pathology, UCSF, in whose laboratory the manuscript was completed.
}

Correspondence should be addressed to Curtis M. Loer, Department of Biology, Lafayette College, Easton, PA 18042-1778.

Copyright $(\mathcal{C} 1993$ Society for Neuroscience $0270-6474 / 93 / 135407-11 \$ 05.00 / 0$ of a neurotransmitter should identify regulatory genes that specify the expression of a given neurotransmitter phenotype. Furthermore, examining phenotypes associated with lack of proper production or handling of a neurotransmitter may reveal interactions dependent on functioning neurons (e.g., Greenspan et al., 1980). By first defining a behavior requiring specific neurons, mutants lacking function of those neurons can be isolated by screening for animals that are defective in that behavior. This approach has proved valuable for isolating mutants defective in the specification and function of the HSN (hermaphroditespccific ncuron), rcquired for cgg laying (Trent et al., 1983; Desai et al., 1988); for mechanosensory neurons (Chalfie and Sulston, 1981); and for GABA-immunoreactive neurons required for defecation (Thomas, 1990) in the free-living nematode Caenorhabditis elegans.

The $C$. elegans adult male contains many sex-specific neurons presumed to function in male mating behavior (Sulston et al., 1980); among these are a number of serotonin-immunoreactive (IR) neurons. Here we define a behavior used in male mating that requires specific serotonin-IR motoneurons and the muscles they innervate. We show that males lacking serotonin are defective in tail curling or "turning" behavior during mating, similar to animals in which these serotonin-IR motoneurons have been ablated. Three serotonin-deficient mutants we examined had this and other behavioral abnormalities in common, suggesting that we may be able to isolate additional serotonindeficient mutants by appropriate behavioral screens. Our initial analysis of these mutants indicates that they are blocked for serotonin synthesis at different steps. Serotonin immunoreactivity in cat -1 and cat-4 mutants was restored by exogenous serotonin or its immediate precursor, whereas bas-1 mutants were only rescued by serotonin. Analysis of these and additional serotonin-deficient mutants may reveal how the expression of a serotonergic phenotype is regulated.

\section{Materials and Methods}

Routine culturing of Caenorhabditis elegans was performed as described by Brenner (1974). Animals were raised at $20^{\circ} \mathrm{C}$, but behavioral observations were made at room temperature, $22-23^{\circ} \mathrm{C}$. Nomenclature used here for $C$. elegans genetics conforms to the conventions set forth by Horvitz et al. (1979).

Strains. The following alleles were used. LGI: unc-29(e1072); LGII: cat-2(e1112); LGIII: dpy-17(e164), unc-32(e189), bas-1(ad446), unc49(e382), lin-39(mu26), lin-39(n1760), mab-5(el239), egl-5(n945); LGIV: lin-22(mu2); LGV: cat-4(e1141), him-5(e1490), unc-5I(e369); LGX: cat-1(ell11). The new mutation bas-1(ad446) (biogenic amine synthesis abnormal) was isolated from the strain MT3239 (a double mutant originally isolated by G. Garriga in a screen for egg-layingdefective mutants), the genotype of which is bas-1(ad446)III; egl(n1397)X. The mutation lin-39(mu26) was isolated by J. Austin and C. Kenyon; the lin-39(mu26); him-5(e1490) strain was generated by S. 

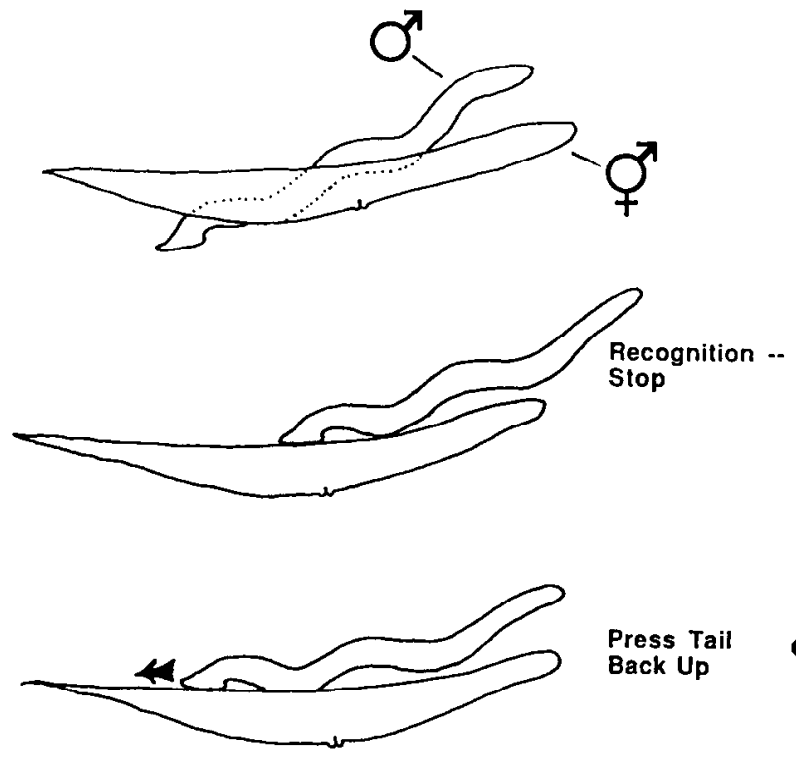

Press Tail Back Up

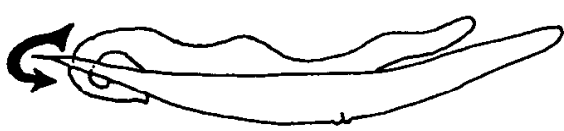

Turn

Repeat until

Vulva is located

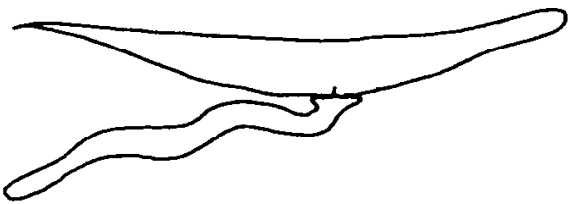

nsert Spicules Transfer Sperm

Figure 1. Steps in male mating behavior. This schematic shows a typical sequence of actions performed by the male following an encounter with a hermaphrodite. When the ventral surface of a male's tail contacts the surface of the hermaphrodite, he stops, presses his tail against the hermaphrodite, and begins to back up. Upon reaching an end of the hermaphrodite, he makes a tight ventral-inward curl of his tail and thereby continues backing up over the opposite surface of his intended mate. This turning behavior may be repeated many times before the vulva is located and copulation initiated.

Salser; this strain was used for mating assays. Since most of the mutants described here have an him-5 mutation in their background (see below), we use him-5 males here as controls and "wild type" in mating, tail curling, and serotonin staining experiments. The him-5 mutants mate normally and have wild-type serotonin immunoreactivity.

Males were generated by four methods: (1) by him-5(e1490) (him: high incidence of males) strains, which generate approximately $30 \%$ males by increased $\mathrm{X}$ chromosome nondisjunction (Hodgkin et al., 1979); (2) by a $6 \mathrm{hr}$ heat shock of L4 (larval stage 4) hermaphrodites at $30^{\circ} \mathrm{C}$ (Sulston and Hodgkin, 1988) (this treatment increases the frequency of male progeny from about $0.2 \%$ to $2-6 \%$ ); (3) in the case of $\mathrm{X}$-linked genes, by mating with wild-type males; (4) by maintenance of a male mating strain. Strains with poor male mating efficiency were not maintained in this manner, because of the possibility of selecting for a strain with improved mating. Males with the mutations lin-39, unc-29, unc- 49 , and bas- 1 were generated by method 1, cat -2 by methods 2 and 4 , cat -1 by method 3 , and cat -4 by methods 1 and 2 . Mating data from males generated by more than one method were compared and found not to be different, so were pooled.

Genetic mapping. The mutation bas-1(ad446) was initially mapped to LGIII using polymorphic sequence-tagged sites detected by polymerase chain reaction (PCR) (Williams et al., 1992). bas-1/+ males were crossed to RW7000 hermaphrodites and hermaphrodite progeny from this cross were cloned. Heterozygous F1 plates carrying bas- 1 were identified by staining progeny with serotonin antisera to identify Bas (serotonin-deficient) worms. F2 progeny from a bas-I-carrying plate were cloned, and allowed to produce progeny, which we stained with serotonin antisera. Six homozygous bas- 1 clones were identified; DNA was made from these and PCR performed. Five RW7000 polymorphisms (stP19, stP120, mgP21, stP127, stP17) located on LGIII were absent in five of six bas- 1 clones; one bas- 1 clone contained the leftmost "stP19" polymorphism alone, presumably due to a recombination even between stP19 and StP120. bas- 1 was subsequently mapped between the genes $d p y-17(e 164) I I I$ and $u n c-32(e 189) I I I$ by standard three-factor mapping (Brenner, 1974). Unc non-Dpy and Dpy non-Unc progeny of dpy-17 unc-32/bas-1 heterozygotes were picked; recombinant chromosomes were made homozygous and clones were stained with serotonin antisera. Phenotypes of 21 homozygous recombinant clones were as follows: 2 Dpy Bas, 3 Dpy, 6 Unc, 10 Bas Unc.

Ablations. Cells were ablated with a focused laser microbeam from a VSL 334 nitrogen-pumped dye laser as previously described (Waring and Kenyon, 1990). Briefly, worms (him-5 males) were anesthetized on agarose pads containing 1-5 mm sodium azide (higher concentrations were used for older animals), and 50-100 pulses of the laser microbeam were delivered to a selected nucleus, until the cell became refractile. In some cases, the worms were checked after a few hours, confirming the death of the selected cell(s). The $\mathrm{M}$ mesoblast was ablated during larval stage 1 (L1), before its division. Success of this ablation could easily be confirmed in the adult male by the presence of crumpled spicules, the result of loss of muscles required for the formation of these structures. The immediate precursors of CP neurons (Pn.aap), which divide in late L3, were ablated in the late L2 or early L3. CP neurons, Pn.aapp, were ablated in the early L4. Unoperated control males (anesthetized and present on the same slide as males receiving laser ablations) were tested and compared with laser-ablated males. These controls were no different from untreated males.

Scoring male behaviors. Tail curling behavior was scored in the following way: adult male worms in M9 buffer (Sulston and Hodgkin, 1988) containing various concentrations of serotonin or other drugs were observed individually through a dissecting microscope for $10 \mathrm{sec}$ each. Males with the tail tightly curled ventrally for $\geq 5 \mathrm{sec}$ were scored as "curled."

Males to be scored for mating behavior were picked at $\mathrm{L} 4$, moved to new plates with bacteria, and scored the next day; all adult males were scored within $1 \mathrm{~d}$ of their final molt. All observations of mating behavior were made blindly; that is, males were placed on coded plates by someone other than the observer. The only exception to blind observations was with $\mathbf{M}$-ablated males; crumpled spicules caused by $\mathbf{M}$ ablation made such males obvious to the observer. An individual adult male was placed on a tiny spot of bacteria (OP50) with four adult unc-51 (paralyzed) hermaphrodites. The small patch of food (bacteria) encouraged the worms to remain within a restricted region of the plate. Each such plate was observed for $5 \mathrm{~min}$, beginning when the male's tail first contacted a prospective mate. Observations were temporarily suspended if a male copulated or left the spot of bacteria.

A turn or attempted turn was scored when the malc backed up to the end (head or tail) of a hermaphrodite and attempted to curl his tail to contact the opposite surface of the mate. Each such turn was placed in one of three categories: good, sloppy, or missed (an example of each is illustrated in Fig. 4). A "good" turn occurred when the male tail remained in contact with the hermaphrodite throughout the turn and continued backing on her opposite side after the turn was completed. A "sloppy" turn occurred when the male's tail temporarily lost contact, but regained contact on the opposite side quickly because of a favorable trajectory (weak or late curling occurred, but was sufficient). A "missed" turn occurred when the male's tail sailed off the end of the hermaphrodite, completely losing contact. Males that made fewer than three turns in 5 min $(5-16 \%)$ were present in all pools and were excluded from quantitative results.

Statistical comparisons of mating assay data were madc by one-factor ANOVA, comparing percentages of good turns among the samples; planned pairwise comparisons were made with Scheffe's $F$ test (Sokal and Rohlf, 1981). We used Hartley's $F$-max test to show that the variances of the samples were not significantly different from one another $(p>0.05)$; therefore, ANOVA was an appropriate means of testing these data (Sokal and Rohlf, 1981) 
A

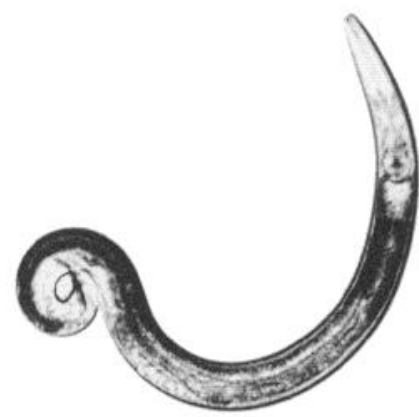

ـ

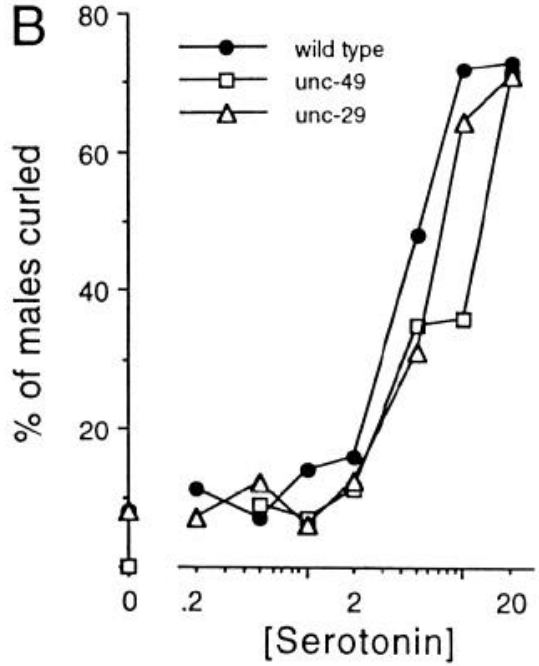

Figure 2. Male tail curling in serotonin. $A$, Lateral view of a normal male in $20 \mathrm{~mm}$ serotonin. Note the tight ventral-inward curl of the tail. The head is up and to the right. Scale bar, $100 \mu \mathrm{m}$. $B$, Tail curling as a function of serotonin concentration. The $y$-axis indicates the percentage of males curled $>50 \%$ of the time plotted against the concentration of serotonin (mM) in M9 buffer (see Materials and Methods). Points at zero serotonin are shown on the y-axis. Number of worms per point: wild type, 43-108, total $n=523 ;$ unc-29, 17-70, total $n=305 ;$ unc-49, 11-28, total $n=$ 138. Solid circles, wild type; open circles, unc-29; triangles, unc-49.
Staining with serotonin antisera. Rabbit antisera to serotonin (paraformaldehyde-conjugated to bovine serum albumin) were purchased from Dr. Henry Steinbusch, Free University, Amsterdam (Steinbusch et al., 1988). The fixation and staining procedure was that of Desai et al. (1988), with slight modifications. Briefly, worms were fixed overnight $(\mathrm{ON})$ at $4^{\circ} \mathrm{C}$ in $4 \%$ paraformaldehyde with or without $1.5 \%$ picric acid (Stefanini et al., 1967); rinsed in 0.5\% Triton X-100/PBS (TrPBS); incubated $\mathrm{ON}$ at $37^{\circ} \mathrm{C}$ in $5 \% \beta-\mathrm{ME}, 1 \%$ Triton $\mathrm{X}-100,0.1 \mathrm{M}$ Tris, $\mathrm{pH}$ 7.4; rinsed in TrPBS; incubated for $2-8 \mathrm{hr}$ at $37^{\circ} \mathrm{C}$ in $2000 \mathrm{U} / \mathrm{ml}$ collagenase type IV (Sigma) in $1 \mathrm{~mm} \mathrm{CaCl}, 1 \%$ Triton X-100, $0.1 \mathrm{~m}$ Tris, $\mathrm{pH} 7.4$; and then rinsed in TrPBS. The worms were "blocked" for $1 \mathrm{hr}$ at room temperature in $1 \% \mathrm{BSA} / \mathrm{TrPBS}$, and then incubated $\mathrm{ON}$ at room temperature in 1:100 anti-serotonin serum in 1\% BSA/TrPBS. The worms were rinsed for $1 \mathrm{hr}$ in $0.1 \% \mathrm{BSA} / \mathrm{TrPBS}$ and incubated for $2-4 \mathrm{hr}$ at $37^{\circ} \mathrm{C}$ with $1: 100$ rhodamine-conjugated goat anti-rabbit IgG (Cappel), rinsed briefly several times in $0.1 \%$ BSA/TrPBS, mounted, and viewed with epifluorescence. Large batches of worms were processed in $1.5 \mathrm{ml}$ microfuge tubes. Single male worms stained following laser ablations were processed in individual wells of multiwell plates and observed during all solution changes. During all antibody incubations of single worms, equivalently fixed and permeabilized broken worms were added to reduce nonspecific staining.

Pharmacological agents. Serotonin (creatinine sulfate complex), 5-hydroxytryptophan (5-HTP), tryptamine, L-dopa, dopamine, tyramine, imipramine, and levamisole were obtained from Sigma. For tail curling assays, drugs were dissolved in M9 buffer. Prior to antibody staining experiments or mating assays, drugs were dissolved in melted NG agar and poured into petri plates. The plates were allowed to cool to room temperature and then used immediately. Worms were placed on drug-containing agar plates for $1-2 \mathrm{hr}$ at $20^{\circ} \mathrm{C}$ before rinsing and fixation (immunostaining experiments) or before being transferred to drug-free plates immediately prior to behavioral testing.

\section{Results}

Mating behavior of the adult male is among the most complex behaviors exhibited by $C$. elegans. The behavior can be divided into at least five components: hermaphrodite recognition, backing, turning, vulval location, and copulation, including spicule insertion and sperm transfer (Fig. 1). When an adult male contacts a hermaphrodite with his tail, he immediately stops, presses his tail firmly against the hermaphrodite, and backs up, moving his tail over the surface of the hermaphrodite. When he reaches the end of his intended mate (at the head or tail), he makes a tight ventral-inward curl to place his tail in contact with the opposite surface. The male then continues moving his tail along that surface in search of the vulval opening. This ventral curling or "turning" behavior may be repeated dozens of times before the vulva is located and copulation is initiated. Thus, male turning behavior, although not absolutely required for copulation, is probably essential for efficient mating.

It is known that $C$. elegans males respond to serotonin (5hydroxytryptamine) by tightly curling the tail and posterior body ventral-inward, similar to tail curling exhibited by males during mating behavior (M. Finney, G. Garriga, and R. Horvitz, personal communication). Croll (1975) also previously noted rapid ventral tail flexions of the related free-living nematode Panagrellus in serotonin-containing solutions. We confirmed and quantified male tail curling in solutions containing serotonin or other drugs (Fig. 2A,B). Serotonin-induced curling was male specific; hermaphrodites did not curl ventrally in serotonin. At higher concentrations (10-20 mM), serotonin paralyzed some worms; many males became immobilized with the tail curled ventral-inward. Tail curling could also be elicited by the application of the related substances tryptamine and 5-HTP. Tryptamine was about as effective at eliciting tail curling as serotonin. In the case of 5-HTP, however, maximal tail curling was only achieved after $30 \mathrm{~min}$ of exposure, in contrast to a few minutes when either serotonin or tryptamine was applied. Tail curling was not elicited by application of dopamine, $\mathrm{L}$-dopa, or tyramine at concentrations up to $20 \mathrm{~mm}$.

Serotonin may elicit tail curling independent of cholinergic and GABAergic neurons, which are believed to mediate excitatory and inhibitory (respectively) control of body wall muscles underlying routine locomotion in C. elegans (Stretton et al., 1985; Chalfie and White, 1988). Exposure to the cholinergic agonist levamisole resulted in a different posture-male worms exhibited a straight shortened body with a slight dorsalward curl of the tail. Males mutant in the gene $u n c-29$, which lack a levamisole receptor (Lewis et al., 1987) and are severely uncoordinated, still exhibited tight tail curling in the presence of serotonin (Fig. 2B). We also tested worms mutant in the gene unc-49, in which GABA binding is substantially reduced (Mancillas et al., 1991); behavior of unc-49 worms is consistent with a loss of GABAergic transmission (Chalfie and White, 1988). These males also displayed serotonin-mediated tail curling at similar levels to wild type (Fig. 2B). Together these results suggest that serotonin acts independently of cholinergic and GABAergic transmission, perhaps by directly causing rapid contraction of specific male muscles. 


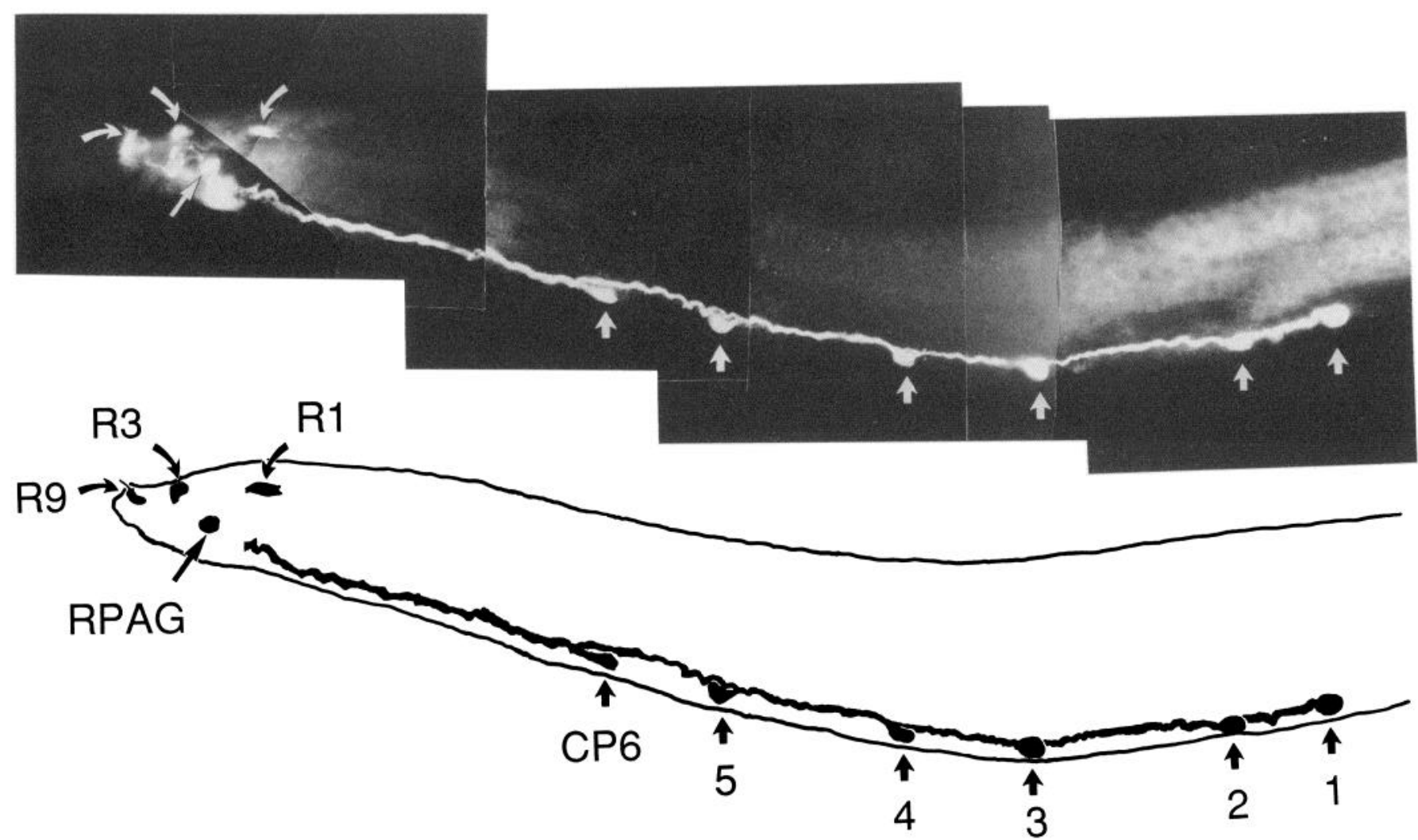

Figure 3. Serotonin-IR neurons specific to the C. elegans male. Above, Photomontage showing CP motoneurons in the male ventral nerve cord, and ray neuron somata, an unpaired soma, and extensive serotonin-IR arborization in the tail stained with anti-serotonin and a rhodamineconjugated secondary antibody. Neuronal somata are labeled with arrows identical to those below. Right lateral view of an adult male posterior; dorsal is up and anterior to the right. Below. Tracing of above preparation identifying neurons labeled with arrows. Thick solid line shows outline of the worm. Curved arrows labeled $R 1, R 3, R 9$, somata of neurons associated with sensory rays 1,3 , and 9 . Long straight arrow labeled RPAG, soma of unpaired neuron found in the right preanal ganglion. Position of the soma and a single ablation experiment suggest that this neuron is either PDC (P11.papa) or PGA (P11.papp), derived from the neuroblast P11.p. Bright staining just anterior and ventral to this soma is an extensive serotonin-IR arborization in the preanal ganglion. Short arrows labeled CP6, 5, 4, 3, 2, 1, somata of CP neurons of the indicated number. The CP neuron (Pn.aapp) is named as the posterior daughter of the neuroblast in the "C" position of the Pn.a neuroblast lineage.

\section{Serotonin-immunoreactive neurons in the male}

The ventral nerve cord of the adult contains six strongly serotonin-IR cell bodies, each of which appears to project a single axon posteriorly (Fig. 3). It has previously been suggested that these somata belong to CP motoneurons (C. Desai and S. McIntire, personal communication; cited by Ellis, 1985) derived from the postembryonic neuroblasts $\mathrm{P}(3-8)$.a (Sulston et al., 1980). Serial section electron microscopic reconstruction of the male ventral nerve cord containing CP neurons derived from $\mathrm{P}(6-9)$.a shows that these neurons synapse on male-specific diagonal muscles (White, 1988); these muscles are appropriately situated to curl the tail when they contract. Thus, CP neurons are good candidates for effectors of male diagonal muscles to cause ventral curling during mating. We confirmed the identity of these neurons by ablating them or their precursors and subsequently staining with serotonin antisera (Table 1).

The male worm contains additional serotonin-IR neurons not found in the hermaphrodite (Chalfie and White, 1988), including a single unpaired neuron in the right preanal ganglion and at least three pairs of neurons apparently associated with the rays, which are sensory structures located in the male tail (Fig. 3). The male tail contains nine bilateral pairs of rays, each consisting of two ciliated neurons and a ray structural cell (Sulston et al., 1980). We identified which rays contain a serotonin-IR neuron by neuronal cell body position and the occasional appearance of a serotonin-IR process within a specific ray. Although processes were only rarely visible in the rays, serotonin-IR processes were seen associated with rays 1,3 , and 9 in more than 10 cases for each ray. Such processes were not seen in other rays. The identification of these rays was further supported by the presence or absence of serotonin-IR somata in mutant backgrounds that delete or alter the position of particular rays (Table 2). Finally, occasionally we saw four additional ventral nerve cord somata located in the central body region and "commissures" to the dorsal nerve cord in the posterior body. These faintly staining somata and processes may belong to CA neurons, sisters of the $\mathrm{CP}$ neurons, which send processes to the dorsal nerve cord to synapse on dorsal muscles (White, 1988).

\section{Effect of ablations of male-specific muscles and neurons on male tail curling}

We eliminated male diagonal muscles by laser ablation of their precursor, the $\mathrm{M}$ mesoblast, which gives rise to diagonal and other postembryonic muscles. Males lacking these muscles no longer made a tight ventral curl in serotonin, confirming that the main mediators of this tail curling response are male-specific, postembryonic muscles. Males lacking diagonal muscles still exhibited a looser ventral curl of the posterior body in serotonin. In M-ablated males, serotonin must be acting on 
Table 1. Serotonin-IR neurons in the male ventral nerve cord

\begin{tabular}{|c|c|c|c|c|c|c|c|}
\hline \multirow[b]{2}{*}{ Cell(s) ablated } & \multicolumn{6}{|c|}{ Pattern of staining somata in VNC } & \multirow[b]{2}{*}{$n$} \\
\hline & $\overline{\mathrm{CP} 1}$ & $\mathrm{CP} 2$ & $\mathrm{CP} 3$ & CP4 & CP5 & $\mathrm{CP6}$ & \\
\hline None & $\mathbf{0}$ & - & 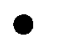 & 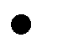 & $\bullet$ & - & Thousands \\
\hline \multicolumn{8}{|l|}{ L3 ablations } \\
\hline$P(3,4)$.aap & - & - & - & - & - & $\bullet$ & 2 \\
\hline$P(5,6)$.aap & 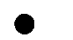 & ? & - & - & - & $\bullet$ & 2 \\
\hline$P(5,7,8)$.aap & - & $\mathbf{0}$ & - & - & - & - & 1 \\
\hline$P(7,8)$.aap & - & $\bullet$ & $\bullet$ & - & - & - & 5 \\
\hline$P(3-8)$. aap & - & - & - & - & - & - & 2 \\
\hline \multicolumn{8}{|l|}{ L4 ablations } \\
\hline$P(7,8)$.aapp & 0 & 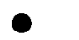 & 9 & - & - & - & 2 \\
\hline$P(3-7)$.aapp & - & - & - & - & - & $\bullet$ & 1 \\
\hline$P(3-8)$.aapp & - & - & - & - & - & - & 3 \\
\hline $\mathrm{P}(3-8) \cdot$ aapp $^{a}$ & - & - & - & - & - & - & 1 \\
\hline $\mathrm{P}(3-8) \cdot$ aapp $^{\alpha}$ & - & $\bullet$ & - & - & - & - & 1 \\
\hline
\end{tabular}

Data represent pattern of serotonin-IR somata in ventral nerve cords (VNC) of normal adult males and adult males that had received specified ablations; anterior is to the left. - indicates the presence of a soma; - indicates the absence of a soma in that position. CP1 (P3.aapp) is the posterior daughter of P3.aap. Ablations done during L3 (larval stage 3) were of CP precursors (Pn.aap); those done during $\mathrm{L} 4$ were of their posterior daughters (Pn.aapp). The number of individuals in which the pattern was observed is indicated at right $(n)$.

${ }^{a}$ In these individuals, either the laser ablation failed to kill the selected cell or the incorrect cell was ablated. $C P 1$ and $C P 2$ neurons [P(3-4).aapp] were typically more difficult to identify than the other CP neurons in $\mathrm{L} 4$ males.

embryonically derived body wall muscles common to males and hermaphrodites. Since hermaphrodites do not curl ventrally in serotonin, these body wall muscles must respond to serotonin differently in the male.

We also observed mating behavior of male worms following ablation of male-specific neurons or muscles. In experiments that were performed blindly, we evaluated the ability of individual male worms to "turn" at the end of a hermaphrodite by means of a tight ventral curl of their tail. In order to reduce variability introduced by movement of the hermaphrodite, we used paralyzed (unc-5l) hermaphrodites for these mating assays. After initial obscrvations, we defined three types of turns: "good," "sloppy," and "missed" (defined in Materials and Methods); each turn observed in subsequent mating assays was classified as one of these three. An example of each type of turn, traced from video recordings, is shown in Figure 4. Ablation of the $\mathrm{M}$ mesoblast, eliminating the development of diagonal and other postembryonic muscles, resulted in males that were severely deficient in turning behavior (Fig. 5). Males with $\mathrm{M}$ ablations displayed mostly "sloppy" turns, during which the slight ventral curl of the posterior body was sufficient to bring the tail back to the opposite side of the hermaphrodite following a brief loss of contact. Many turns were completely unsuccessful or "missed." These males never made normal turns, although occasionally a male was able to make an abnormal "good" turn by pivoting his entire body about his slightly curled tail. This pivoting behavior was exhibited only rarely by unoperated males.

Ablation of CP neurons or their immediate precursors also disrupted the ability of males to make a tight ventral curl of the tail during turning, although they were not as severely affected as M-ablated males (Fig. 5). Ablation of the immediate precursors of the $\mathrm{CP}$ and $\mathrm{CA}$ motoneurons, $\mathrm{P}(3-8)$.aap, yielded males that were able to make a normal good turn about one-third of the time. Males in which CP neurons alone [P(3-8).aapp] were ablated were perhaps slightly better at turning, although the difference was not statistically significant. We also tested males in which we ablated between one and four CP neurons or their precursors. The turning ability of males with two or more $\mathrm{CP}$ neurons remaining was not significantly different from that of control males, suggesting that $\mathrm{CP}$ function is normally redundant or that the remaining neurons compensate for loss of other CP neurons. This result also indicates that the disruption of turning behavior by $\mathrm{CP}$ ablation is unlikely to be caused by nonspecific effects of laser ablation.

Finally, we tested the turning ability of males carrying the mutation lin-39. In lin-39 hermaphrodites, P3-8.aap die (Ellis, 1985; Horvitz, 1988). Although these cells do not appear to die in lin-39 males (Ellis, 1985), we found that lin-39 males completely lacked serotonin-IR somata and processes in the ventral nerve cord in two different lin-39 alleles ( $m u 26, n=93 ; n 1760$, $n=79$ ). These mutant males appeared to possess a normal complement of serotonin-IR neurons in the head and tail. We tested mating of males carrying one of these alleles, lin-39(mu26), which molecular analysis shows is a null allele (Wang et al., 1993). The turning ability of these lin-39 males was similar to that of males with CP ablations (Fig. 5).

\section{Turning ability of serotonin-deficient mutants}

If tail curling is caused by CP neurons releasing serotonin on male diagonal muscles, then mutations that reduce or eliminate the expression of serotonin should result in mutant males that

Table 2. Serotonin-IR neurons in the male tail

$\begin{array}{lll}\text { Genotype } & \text { Alteration of ray pattern } & \text { Pattern of } \\ \text { Wild type } & \text { None } & \text { serotonin-IR somata } \\ \text { mab-5 } & \text { Rays 1-6 missing } & 3 \text { present (rays 1, 3,9) } \\ e g l-5 & \text { Ray 6 missing } & 2 \text { anterior somata missing } \\ l i n-22 & \text { Additional ray 1-like } & 3 \text { present } \\ & \text { structures along body } & 3 \text { normal present plus extra } \\ \text { pal-l } & \text { Ray 1 displaced anteriorly, rays } & \text { somata along body } \\ & 2-6 \text { missing } & 1 \text { soma displaced anteriorly, } \\ \end{array}$

Data are patterns of serotonin-IR somata found in tails of wild-type males and mutant males with altered patterns of sensory rays. The observations are consistent with autonomous expression of a serotonergic phenotype among the remaining ray neurons in these mutants.

a References for altered ray patterns are as follows: mab-5, Kenyon (1986); egl-5, Chisholm (1991); lin-22, Horvitz et al. (1983), Waring et al. (1992); pal-1, Waring and Kenyon (1990). 


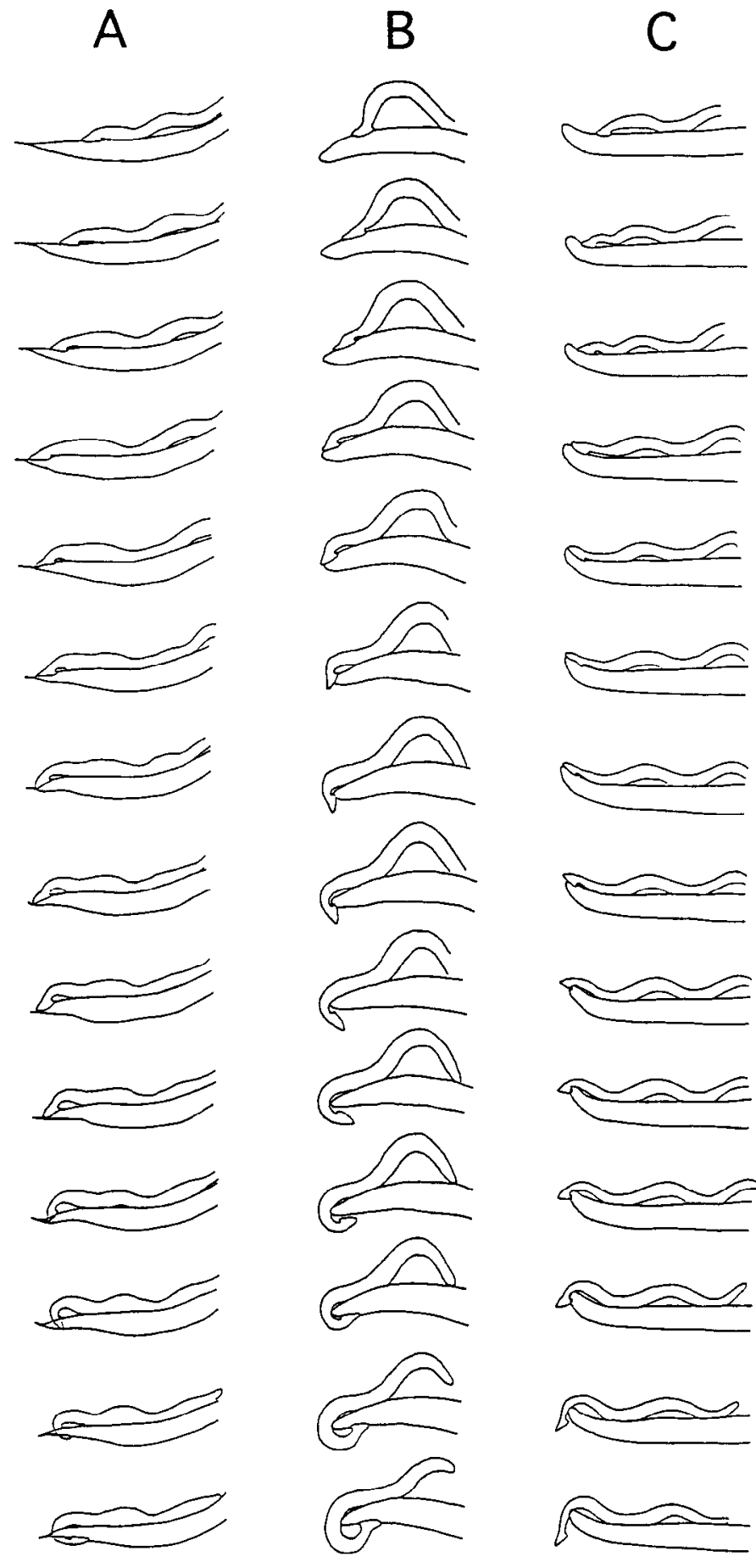

Figure 4. Male turning behavior. Selected frames traced from video recordings progress in time from top to bottom. Frames shown are in correct sequence but are not necessarily consecutive. The total elapsed time for each sequence is approximately $2 \mathrm{sec} . A$, "Good" turn, wildtype male. $B$, "Sloppy" turn, wild-type male. $C$, "Missed" turn, cat-4 male.

are unable to curl their tails during mating. We tested two mutants that have previously been described to be deficient in serotonin (Desai et al., 1988) and one previously uncharacterized mutant; these mutants define three genetic loci, each represented by a single recessive allele. Worms with the mutation cat-1(e1111)I have variably reduced serotonin-IR; they range from fully wild-typc staining to a complcte lack of staining.
Worms with the mutation cat-4(e1141) $\mathrm{V}$ completely lack serotonin-IR. Both cat-1 and cat-4 worms appear superficially wild type in movement and other behaviors, although they have previously been shown to be poor at siring offspring (Hodgkin, 1983), suggesting a defect in mating behavior. When cat-1 males were tested in our mating behavior assay, they showed variable reduction in their turning ability (Fig. 6), consistent with their variable ability to make serotonin, although we did not stain and compare individual cat-1 male worms. Some cat-1 males were "perfect," making $100 \%$ good turns. Males carrying cat-4 were more severely defective in turning ability, frequently making "missed" turns, as illustrated by a sequence traced from video recordings (Fig. $4 C$ ). Worms carrying the previously undescribed mutation bas-1(ad446)III (kindly sent to us by $\mathrm{G}$. Garriga) had no immunocytochemically detectable serotonin, like cat-4 worms. bas- 1 males were similar to cat-4 males in their poor turning ability (Fig. 6). Although cat-4 and bas-1 males made some good turns, no such males were perfect, making all good turns. These worms demonstrated turning ability similar in quality to that shown by male worms in which $\mathrm{CP}$ neurons were ablated.

Both cat-1 and cat-4 worms are not only serotonin deficient, but also have altered dopamine expression; they were originally isolated on this basis (Sulston et al., 1975). We have not examined the expression of dopamine in bas-1 worms. To test whether turning defects in cat -1 and cat -4 might be caused by lack of dopamine, we tested males carrying the mutation cat2(e1112)II, which are dopamine deficient, but have normal serotonin immunoreactivity. These male worms were close to wild type in their turning ability (Fig. 6), suggesting that serotonin deficiency, and not dopamine deficiency, is the cause of the turning disability in cat -1 and cat -4 males.

In addition to their poor male turning ability, these three mutants (cat-1, cat-4, and bas-1) shared other subtle behavioral abnormalities that we did not quantify. These traits were perhaps less pronounced in cat- $l$ animals. Mutant males often seemed unable to press the surface of the hermaphrodite as firmly as wild-type males, "tracking" poorly; sometimes they lost contact with the hermaphrodite while backing up. These males occasionally made rapid dorsal-inward curls of the tail and posterior body as they backed up along a hermaphrodite, opposite to the serotonin-induced ventralward curl. Sometimes following such dorsal curls or following missed turns, a mutant male coiled his entire length tightly dorsal-inward. Such a coiled male could remain in this posture for many seconds-seeming to have difficulty in escaping from it-repeatedly returning to a tight dorsal-inward coil after brief partial relaxations. Such dorsal-inward coils also seemed to occur spontaneously. This dorsal-inward posture was rarely adopted by wild-type males, and then maintained only briefly. Finally, although their movement was superficially normal, these serotonin-deficient worms appeared sometimes to move about more rapidly than normal.

Serotonin-deficient males may also suffer a sensory deficit caused by lack of serotonin in sensory ray neurons. When a wild-type male contacts a hermaphrodite with his tail, he usually stops, presses, and backs up along the hermaphrodite. cat-1, cat -4 , and bas- 1 males were more likely to ignore contact with a hermaphrodite and continue without stopping or backing. We noted, however, that dopamine-deficient cat-2 males also suffered from a relative lack of interest in hermaphrodites. Three pairs of ray neurons $(5,7$, and 9 ) have previously been shown to contain dopamine (Sulston et al., 1975, 1980). Thus, dopa- 


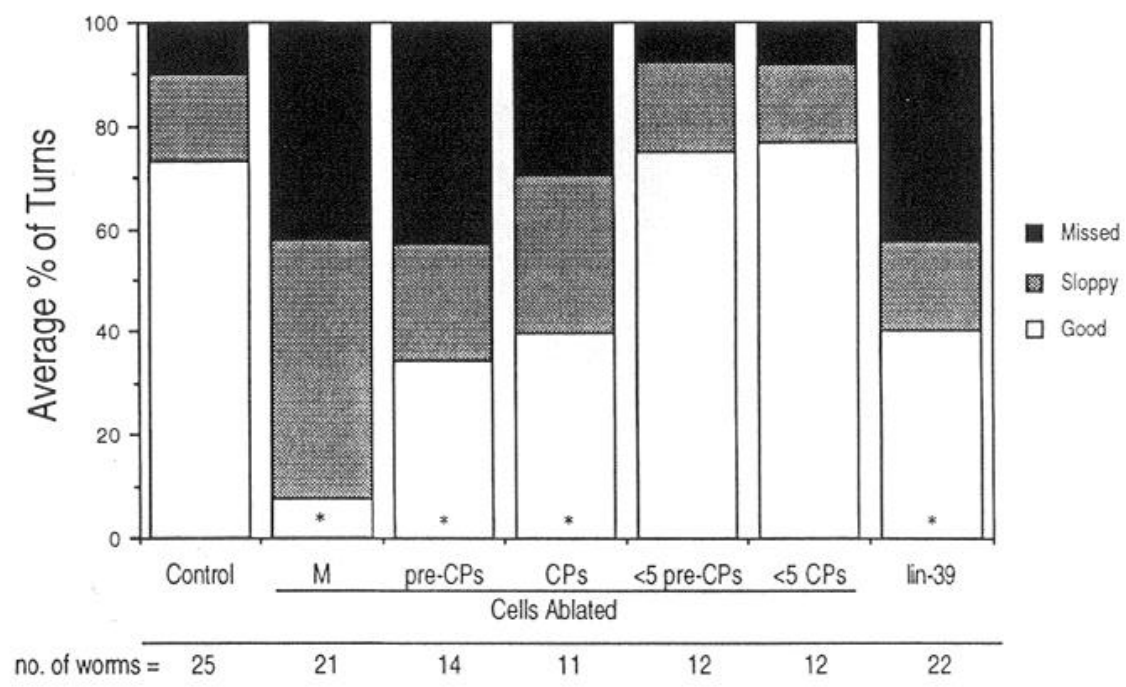

Figure 5. Turning ability of unoperated males, males with the indicated ablations, and lin-39 males. CP and pre-CP (precursor to CP, Pn.aap) ablations include males in which five or six cells were ablated; $\leq 5$ columns indicate males in which one to four cells were ablated. Legend at right indicates percentage of turns that were missed (solid), sloppy (shaded), or good (white). Turning data are represented as the average percentage of turns. The percentage of each type of turn is determined for each worm and averaged by the number of worms. This normalization corrects for the fact that the number of turns scored per worm ranged from 3 to 21 (average, 7.5 turns per worm). Analysis by ANOVA of the percentages of good turns within each group revealed statistically significant differences among the groups $(p=0.0001)$. Columns marked with asterisks are significantly different $(p<0.05)$ from control and other unmarked columns in pairwise comparisons (Scheffè's $F$ test). Columns with asterisks were not significantly different from one another; likewise, unmarked columns were not significantly different from one another.

mine deficiency may account for some or all of the "disinterest" in hermaphrodites displayed by cat-1, cat-4, and perhaps bas- 1 male worms.

\section{Rescue of serotonin-deficient mutants by serotonin or 5-HTP}

The serotonin-deficient mutants we describe here identify genes that are required for normal expression of a serotonergic phenotype. What aspects of a serotonergic phenotype do the normally serotonin-IR neurons retain despite these mutations? Despite common behavioral traits that may be attributable to the lack of serotonin and/or dopamine, we found that treatment of these mutants with serotonin or its immediate precursor, 5-HTP, suggested they are blocked at different steps of serotonin synthesis or handling (Fig. 7). Worms carrying a mutation in cat-4 completely lacked serotonin immunoreactivity; however, exposure to serotonin or 5-HTP rescued this serotonin deficiency. cat -1 mutants were similarly restored to full serotonin immunoreactivity by treatment with serotonin or 5-HTP. In contrast, the serotonin immunoreactivity of bas- 1 worms was not restored by exposure to 5-HTP, but was restored by exposure to serotonin. (These results with cat-1, cat -4 , and bas- 1 were also previously noted independently by G. Garriga and H. R. Horvitz, personal communication.) Figure 8 shows the results of a staining experiment (two experiments are shown for cat-4) in which serotonin-IR of cat-4, bas-1, and wild-type worms was quantified following exposure to a range of concentrations of serotonin and 5-HTP. Rescue of serotonin-IR in cat-4 worms by exposure to 5-HTP was evident over a wide range of concentrations, down to $10 \mu \mathrm{M}$. Exposure to $1-10 \mathrm{~mm}$ 5-HTP rescued nearly all worms; many exhibited normal serotonin-IR in all appropriate neurons. Rescue of staining in cat 4 worms by exposure to serotonin was similar to that seen with 5-HTP, but more variable, especially at higher concentrations. Exposure to 2-10 mM serotonin typically decreased or eliminated serotoninIR in neurons of wild-type or mutant worms and greatly in- creased background staining throughout the animal. Simultaneous exposure to imipramine (a serotonin uptake inhibitor) completely blocked the rescue of serotonin-IR in cat-4 worms by serotonin and reduced rescue by 5 -HTP (data not shown).

Exposure to 5-HTP (and to a lesser degree, serotonin) not only loads serotonin-IR neurons, but also causes known dopamine-containing neurons to become serotonin-IR in wildtype worms (Garriga and Horvitz, personal communication). These same dopamine-containing neurons became serotoninIR in 5-HTP-exposed cat-1, cat-2, and cat-4 worms but not in

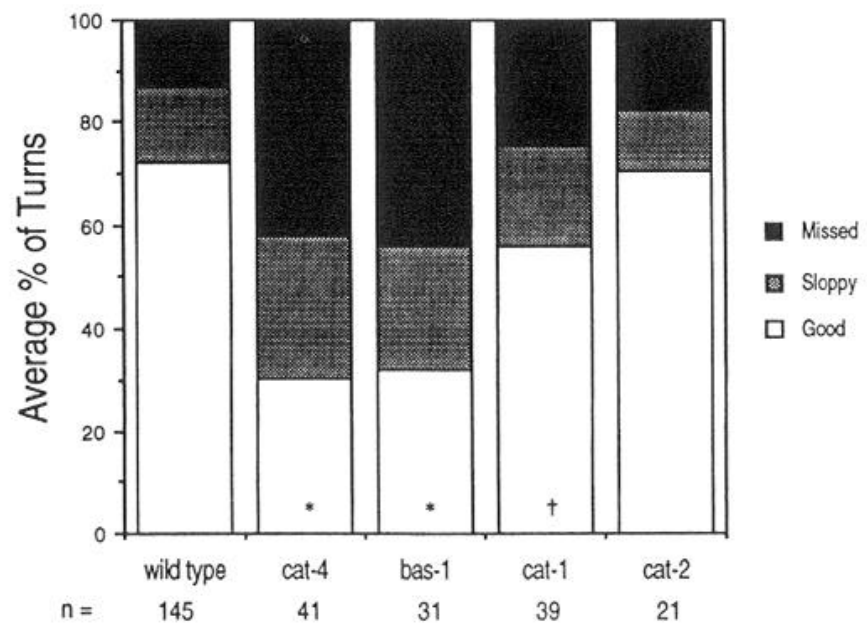

Figure 6. Graph of turning ability of wild-type and cat-4, bas-1, cat1 , and cat-2 mutant males. Data are presented as in Figure 5. Analysis by ANOVA revealed statistically significant differences among the groups $(p=0.0001)$. Columns marked with asterisks are significantly different $(p<0.05)$ from wild type, cat -1 , and cat-2, and not significantly different from one another. cat $-1(\dagger)$ was significantly different from wild type, but not cat-2. Wild type and cat-2 were not significantly different from one another. 


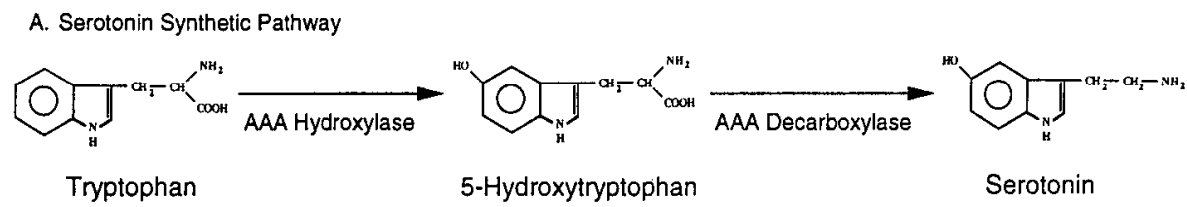

Figure 7. A, Serotonin synthetic pathway. Tryptophan is first hydroxylated to 5-hydroxytryptophan (5-HTP) by an aromatic amino acid $(A A A)$ hydroxylase, and then decarboxylated to yield serotonin (5-HT, 5-hydroxytryptamine) by an aromatic amino acid decarboxylase. $B$, Summary of characteristics of serotonin-deficient mutants.
B. Restoration of Serotonin Immunoreactivity in Mutants

\begin{tabular}{c|c|c|}
\multicolumn{1}{c}{} & \multicolumn{1}{c}{ rescue by 5-HTP? } & rescue by serotonin? \\
\cline { 2 - 3 } cat-1, cat-4 & YES & YES \\
\cline { 2 - 3 } bas-1 & NO & YES \\
\cline { 2 - 3 } & &
\end{tabular}

5-HTP-exposed bas- 1 worms (data not shown). The most reliably staining neurons in wild-type and mutant worms were the deirid and postdeirid neurons (ADE and PDE), followed by four head neurons (CEPs), all common to males and hermaphrodites. Dopamine-containing ray neurons in males were only rarely stained. In many worms, another pair of somata was seen in the tail, sometimes with processes projecting posteriorly into the tail spike. These cells are probably phasmid neurons (Horvitz et al., 1982).

The normal complement of serotonin-IR neurons was apparent in cat -1, cat -4 , and bas -1 worms rescued by exposure to serotonin. Furthermore, the location and extent of serotoninIR processes, including those of the CP neurons, also appeared to be normal in these mutant worms. Thus, the behavioral defects in these mutants are not caused by the loss of normally serotonin-IR neurons or gross disruption of axonal projections. Interestingly, Drosophila mutants $(D d c)$ lacking serotonin also appear to generate a normal set of neurons that can recover serotonin immunoreactivity by application of exogenous serotonin (Vallés and White, 1986).

Recovery of serotonin immunoreactivity by exposure to 5-HTP or serotonin suggested that the turning disability of serotonindeficient mutants might also be rescued by such treatment. The turning defect of cat-4 males was rescued by a $1 \mathrm{hr}$ exposure to
$1 \mathrm{~mm}$ serotonin (Fig. 9), a treatment that reliably restored serotonin immunoreactivity in cat -4 mutants. This result strongly supports the hypothesis that the turning defect of cat 4 males is caused by a lack of serotonin.

\section{Discussion}

\section{Serotonin as a neurotransmitter in $\mathrm{C}$. elegans and other nematodes}

Serotonin has been detected in free-living and parasitic nematodes by immunocytological and chromatographic methods; it has been suggested to be involved in locomotion, reproductive behaviors, pharyngeal pumping, and even carbohydrate metabolism (Croll, 1975; Donahue et al., 1981; Horvitz et al., 1982; Avery and Horvitz, 1990). In C. elegans and other nematodes, serotonin-IR fibers innervate vulval regions and serotonin can induce egg laying. The serotonin-IR processes associated with vulval muscles in C. elegans belong to the HSN (hermaphroditespecific neuron). When the HSN is ablated, hermaphrodites become severely defective in egg laying (H. R. Horvitz and J. Sulston, unpublished observations; cited by Trent et al., 1983). Surprisingly, serotonin-deficient mutants are not egg laying defective, although residual serotonin may be present, as imipramine (which blocks serotonin uptake) is able to elicit egg laying in these mutants (Desai et al., 1988). It is possible that very
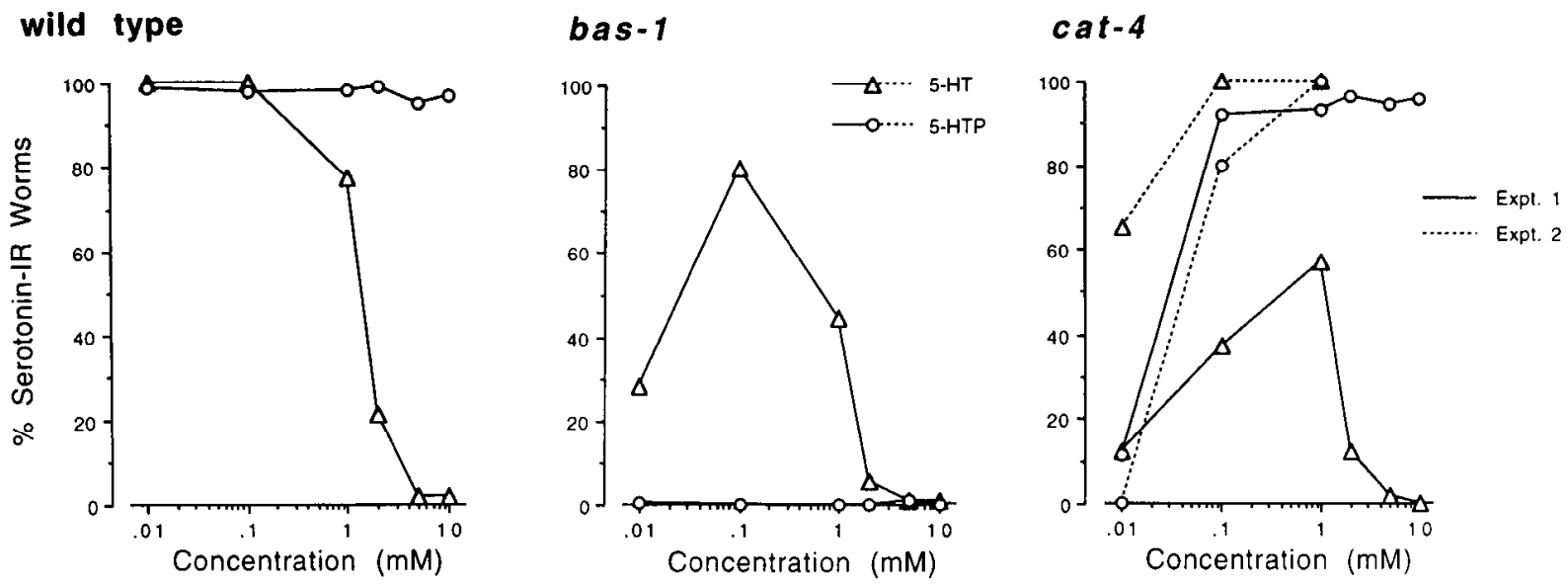

Figure 8. Rescue of serotonin-IR in mutants. Each graph indicates the percentage of worms containing one or more serotonin-IR neuron(s) following exposure to the indicated concentration (mM) of serotonin (triangles) or 5-HTP (circles) in wild-type, bas-1, and cat-4 worms (left to right). With no serotonin or 5-HTP present, $\sim 100 \%$ of wild-type worms are serotonin-IR; $0 \%$ of bas-1 and cat-4 worms are serotonin-IR. Each point represents at least 100 worms. All data points connected with solid lines are from a single experiment (Expt. I). For cat-4, data from a second experiment in which three concentrations were tested are also shown (Expt. 2, dashed lines); these results with serotonin exposure were more typical than those of the first experiment. 


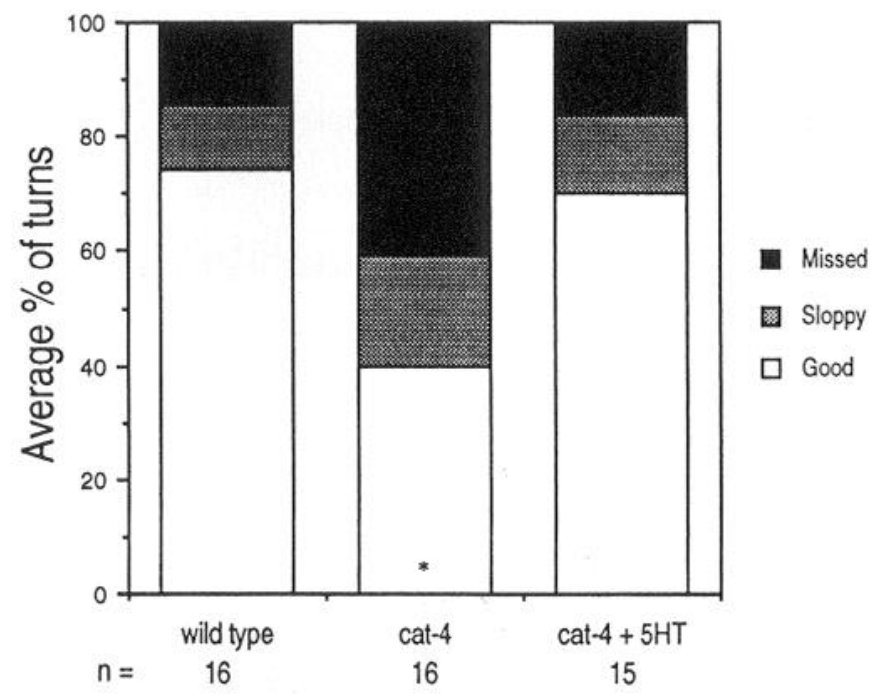

Figure 9. Rescue of turning behavior in cat-4 mutant males by serotonin. Control and experimental worms were scored together under otherwise identical conditions. Data are represented as in Figure 5. Analysis by ANOVA revealed statistically significant differences among the groups $(p=0.002)$. Pairwise comparisons show that cat $-4\left(^{*}\right)$ is significantly different $(p<0.05)$ from wild type and cat-4 + serotonin, which are not different from one another.

little serotonin is required for HSN function or that some other neurotransmitter is present and sufficient for HSN function.

Among the brightest, most reliably staining serotonin-IR somata and processes in $C$. elegans are those belonging to the NSMs (neurosecretory motoneurons) located within the pharynx. These neurons contain large dense-core vesicles characteristic of monoamine-secreting cells (Albertson and Thomson, 1976). Both serotonin and imipramine stimulate pumping (Horvitz et al., 1982). cat-4 mutants display decreased rates of pumping under appropriate conditions; consistent with the presence of residual serotonin in cat-4 mutants, pumping is slightly enhanced when imipramine is applied (Avery and Horvitz, 1990). Ablation of NSMs, however, has no obvious effect on pharyngeal pumping (Avery and Horvitz, 1989) and worms lacking NSMs are still stimulated to pump by imipramine, suggesting regulation of pumping by a different serotonin-IR neuron (D. Raizen, H. Rosenfeldt, and L. Avery, personal communication).

Serotonin-IR processes in posterior ganglia of male C. elegans and other nematodes have suggested male-specific functions for serotonin. We have confirmed previous observations that application of serotonin to male worms results in tail curling reminiscent of that exhibited by males during turning behavior. Although the concentration of serotonin required to elicit tail curling is high (the cuticles of intact nematodes are notoriously impermeable), there is good reason to believe that the effect is specific. Tail curling in $C$. elegans is elicited by serotonin and closely related substances (5-HTP and tryptamine). Dopamine and related substances do not cause tail curling at high concentrations. Treatment with the cholinergic agonist levamisole results in males adopting a different posture, suggesting that male tail curling in serotonin is probably not caused by nonspecific activation of ACh-sensitive body wall muscles. Males of the large parasitic nematode Ascaris, which possess five male-specific serotonin-IR neurons in their ventral nerve cord (Stretton and Johnson, 1985), also exhibit tail curling when relatively low levels of serotonin $(1 \mu \mathrm{M})$ are injected into the posterior body cavity (C. Buchanon and A. Stretton, personal communication).

\section{The CP motoneurons play a role in male turning behavior}

Ablation of male diagonal muscles, the targets of CP neurons, results in males that never make normal turns, whereas males lacking CP neurons are still able to make some good turns. There are a number of possible explanations for this. First, we eliminated diagonal muscles by ablation of the M mesoblast, which also eliminates other postembryonically derived muscles in the male, not just the diagonal muscles. Among these are a small number of additional body wall muscles and other muscles in the male tail. Some of these muscles, not under the control of the $\mathrm{CP}$ neurons, may be partly responsible for curling the tail. Ablation of diagonal muscles alone might cause a less severe defect, resulting in males that turn with proficiency similar to $\mathrm{CP}$-ablated males. Second, it is possible that the CP motoneurons are not the sole activators of diagonal muscles. This seems likely since worms with CP ablations are not completely incapable of making tight, "good" turns, although those they do make are often slower than normal. CP motoneurons may assist in coordination or correct timing of the turn; for example, release of serotonin by the CPs may allow the diagonal muscles to contract rapidly and strongly when required. Serotonin is known to modulate muscle contractility in other invertebrates. In the leech, for example, serotonin increases the rate and amplitude of ACh-induced contraction of body wall muscles (Mason and Kristan, 1982). Serotonin can increase ACh-induced contraction of individual, isolated molluscan muscle fibers (Zoran et al., 1989); in vivo, serotonin has been demonstrated to enhance or inhibit contraction, depending upon the muscle tested (Ram et al., 1981). Serotonin can also modulate neuronal function presynaptically; it increases ACh release at the crayfish neuromuscular junction (Delaney et al., 1991). Our results with the mutants unc-29 and unc- 49 suggest, however, that tail curling by male C. elegans does not require cholinergic or GABAergic transmission believed to control body wall muscle contractions.

If additional ventral nerve cord motoneurons are involved in male turning behavior, their role may be difficult to assess, since they are likely to be the same motoneurons (putatively cholinergic and GABAergic) used for routine locomotion. Killing these neurons would yield a grossly uncoordinated worm, incapable of normal forward and/or backward movement. In contrast, male worms lacking CP neurons move normally forward and backward, suggesting that $\mathrm{CP}$ neurons are mainly required for male mating behavior. As activators of male diagonal muscles, $\mathrm{CP}$ motoneurons may also be important in maintaining antagonism with muscles that flex the posterior body dorsally. Lack of such antagonism may cause the poor tracking, rapid dorsal flexions, and dorsal-inward coiling exhibited by cat-1, cat-4, and bas- 1 males.

Although cat -1 , cat -4 , and bas -1 males may be defective in up to 6 of 18 pairs of ray neurons (three each containing serotonin and dopamine), these mutant worms are no more defective in turning behavior than males in which only the CP neurons have been ablated. Loss of ray sensory function need not be invoked to explain the turning defect in serotonin-deficient males, although it could contribute to this defect. Reduced ray neuron function probably does contribute to the poor recognition of hermaphrodites by cat -1 , cat -4 , and bas- 1 males. Ablation of all rays, or rays 1-6 alone, results in males that have difficulty in recognizing hermaphrodites $(\mathrm{K}$. Liu and P. Sternberg, personal communication). On the other hand, loss of rays 7-9 by ablation of their common precursor results in males that fail to uncurl as they turn, often coiling ventral-inward during an at- 
tempted turn (Liu and Sternberg, personal communication). This phenotype is opposite to that observed in serotonin-deficient mutants.

\section{Serotonin-deficient mutants have different alterations in serotonergic phenotype}

Since staining of normally serotonin-IR neurons in cat-1, cat4 , and bas- 1 mutants can be restored by exogenous serotonin, none of the three mutations we analyzed appears to cause a total loss of "serotonergic identity" in these neurons (Fig. 7). Both cat -1 and cat -4 mutations affect both dopamine and serotonin expression, so their defects must lie in genes required in common among neurons using these neurotransmitters. Sulston et al. (1975) failed to detect changes in tyrosine hydroxylase or dopa decarboxylase activities (required for dopamine synthesis) in cat -1 or cat -4 mutants. The cat -1 mutation results in loss of dopamine in processes, but not somata, and can be phenocopied by reserpine treatment, which in mammals depletes vesicles of monoamines (Sulston et al., 1975). Serotonin immunoreactivity in cat 1 mutants is not obviously reduced in processes more than cell bodies; the effect seems to be a variable overall reduction in staining. Still, the defect in cat-1 mutants could be in a vesicular monoamine transporter used by both types of neurons. Serotonin immunoreactivity could conceivably be restored by 5 -HTP or serotonin in such a mutant.

Although one simple explanation for the defect in cat-4 mutants is an inability to synthesize 5-HTP by loss of a tryptophanhydroxylating enzyme, this alone cannot explain the concurrent reduction in dopamine. A plausible explanation might be a defect in an enzyme required for biosynthesis or recycling of a pterin cofactor used by both dopamine- and serotonin-synthetic hydroxylases in many organisms (e.g., O'Donnell et al., 1989). cat -4 could, of course, be a regulatory gene required by both types of neurons. The ability of serotonin to rescue staining and behavior in cat-4 mutant males suggests that these mutants retain the ability to use the neurotransmitter normally. It is possible, however, that serotonin rescues turning ability by acting directly on diagonal muscles and that normal CP function is impaircd in cat-4 malcs.

Mutation in the bas 1 gene may simply disrupt the enzyme (an aromatic amino acid decarboxylase) required for making serotonin from 5-HTP, since immunoreactivity of bas- $1 \mathrm{mu}$ tants is only restored by serotonin, and not its precursor. In fruit flies and mammals, a single decarboxylase is used for both serotonin and dopamine synthesis (Livingstone and Tempel, 1983; Albert et al., 1987). The observation that known dopamine-containing neurons in the worm become serotonin-IR upon exposure to 5-HTP, and that this property is also lost in bas-1 mutants suggests that this is also true for $C$. elegans. This further suggests that bas-1 mutants are deficient in this activity, whether by mutation of the structural gene itself or another gene that regulates its expression.

Some caution must be exercised in the interpretation of results in which these serotonin-deficient mutants have been treated with serotonin or 5-HTP. Although all threc mutations are rccessive, they do not necessarily represent complete loss (or even reduction) of gene function. For example, if the cat-4 mutation severely decreased the efficiency of a serotonin-synthetic decarboxylase (but did not eliminate it), excess substrate (5-HTP) might serve to restore serotonin synthesis to normal levels. If serotonin and dopamine synthetic pathways shared this decar- boxylase, such a mutation would also lead to a reduction in dopamine expression.

The serotonin-deficient mutants we have analyzed were identified directly - by staining with serotonin antisera-a difficult process to use for screening many animals for new serotonindeficient mutants. The results we report here suggest that we may be able to identify additional mutants by screening for worms that display behavior abnormalities typical of these mutants. Furthermore, as with the mutants described, new mutants should fall into different classes, based on serotonergic markers such as the ability to synthesize serotonin from a precursor or to take up serotonin itself. Further analysis of cat-1, cat-4, bas1 , and newly identified mutants with these and additional serotonergic markers may allow us to learn how the expression of a serotonergic phenotype is controlled.

\section{References}

Albert VR, Allen JM, Joh TH (1987) A single gene codes for aromatic L-amino acid decarboxylase in both neuronal and non-neuronal tissues. J Biol Chem 262:9404-9411.

Albertson D, Thomson J (1976) The pharynx of C. elegans. Philos Trans R Soc Lond [Biol] 275:299-325.

Avery L, Horvitz HR (1989) Pharyngeal pumping continues after laser killing of the pharyngeal nervous system of $C$. elegans. Neuron 3:473485.

Avery L, Horvitz HR (1990) Effects of starvation and neuroactive drugs on feeding in Caenorhabditis elegans. J Exp Zool 253:263-270.

Brenner S (1974) The genetics of C. elegans. Genetics 77:71-94.

Chalfie M, Sulston J (1981) Developmental genetics of the mechanosensory neurons of Caenorhabditis elegans. Dev Biol 82:358-370.

Chalfie M, White J (1988) The nervous system. In: The nematode Caenorhabditis elegans (Wood WB, ed), pp 337-391. Cold Spring Harbor, NY: Cold Spring Harbor Laboratory.

Chisholm A (1991) Control of cell fate in the tail region of C. elegans by the gene egl-5. Development 111:921-932.

Croll $N$ (1975) Indolealkylamines in the coordination of nematode behavioral activities. Can J Zool 53:894-903.

Delaney K, Tank DW, Zucker RS (1991) Presynaptic calcium and serotonin-mediated enhancement of transmitter release at crayfish neuromuscular junction. I Neurosci 11:2631-2643.

Desai C, Garriga G, McIntire SL, Horvitz HR (1988) A genetic pathway for the development of the Caenorhabditis elegans HSN motor neurons. Nature 336:638-646.

Donahue MJ, Yacoub NJ, Michnoff CA, Masaracchia RA, Harris BG (1981) Serotonin (5-hydroxytryptamine): a possible regulator of glycogenolysis in perfused muscle segments of Ascaris suum. Biochem Biophys Res Commun 101:112-117.

Ellis H (1985) Genetic control of programmed cell death in the nematode Caenorhabditis elegans. $\mathrm{PhD}$ thesis, Massachusetts Institute of Technology.

Greenspan RJ, Finn JA, Hall JC (1980) Acetylcholinesterase mutants in Drosophila and their effects on the structure and function of the central nervous system. J Comp Neurol 189:741-774.

Hodgkin I (1983) Male phenotypes and mating efficiency in C. elegans. Genetics 103:43-64.

Hodgkin J, Horvitz H, Brenner S (1979) Nondisjunction mutants of the nematode $C$. elegans. Genetics 91:67-94.

Horvitz H, Brenner S, Hodgkin J, Herman R (1979) A uniform genetic nomenclature for the nematode $C$. elegans. MGG 175:129-133.

Horvitz H, Chalfie M, Trent C, Sulston J, Evans P (1982) Serotonin and octopamine in the nematode C. elegans. Science 216:1012-1014.

Horvitz H, Sternberg P, Greenwald I, Fixsen W, Ellis H (1983) Mutations that affect neural cell lineages and cell fates during the development of the nematode C. elegans. Cold Spring Harbor Symp Quant Biol 48:453-463.

Horvitz HR (1988) Genetics of cell lineage. In: The nematode Caenorhabditis elegans (Wood WB, ed), pp 157-189. Cold Spring Harbor, NY: Cold Spring Harbor I aboratory.

Kenyon C (1986) A gene involved in the development of the posterior body region of $C$. elegans. Cell 46:477-487. 
Lewis JA, Elmer JS, Skimming J, McLafferty S, Fleming J, McGee T (1987) Cholinergic receptor mutants of the nematode Caenorhabditis elegans. J Neurosci 7:3059-3071.

Livingstone MS, Tempel BL (1983) Genetic dissection of monoamine neurotransmitter synthesis in Drosophila. Nature 303:67-70.

Mancillas JR, Ruiz-Morales A, Olsen R (1991) GABA receptor function is altered in the $C$. elegans mutant unc-49. Soc Neurosci Abstr $17: 526$.

Mason A, Kristan WB (1982) Neuronal excitation, inhibition, and modulation of leech longitudinal muscle. J Comp Physiol 190:283302.

O'Donnell JM, McLean JR, Reynolds ER (1989) Molecular and developmental genetics of the Punch locus, a pterin biosynthesis gene in Drosophila melanogaster. Dev Genet 10:273-286.

Ram JL, Shukla UA, Ajimal GS (1981) Serotonin has both excitatory and inhibitory modulatory effects on feeding muscles in Aplysia. J Neurobiol 12:613-621

Sokal RR, Rohlf FJ (1981) Biometry. New York: Freeman.

Stefanini M, DeMartino C, Zamboni L (1967) Fixation of ejaculated spermatozoa for electron microscopy. Nature 216:173-174.

Steinbusch HWM, Wouterlood FG, deVente J, Bol JGJM, Berkenbosch $F$ (1988) Immunohistochemical localization of monoamines and cyclic nucleotides. Acta Histochem S35:85-106.

Stretton AOW, Johnson CD (1985) GABA and 5HT immunoreactive neurons in Ascaris. Soc Neurosci $\Lambda$ bstr 11:626.

Stretton AOW, Davis RE, Angstadt JD, Donmoyer JE, Johnson CD (1985) Neural control of behaviour in Ascaris. Trends Neurosci 8:294300 .

Sulston J, Hodgkin J (1988) Methods. In: The nematode Caenorhabditis elegans (Wood WB, ed), pp 587-605. Cold Spring Harbor, NY: Cold Spring Harbor Laboratory.
Sulston J, Dew M, Brenner S (1975) Dopaminergic neurons in the nematode C. elegans. J Comp Neurol 163:215-226.

Sulston J, Albertson D, Thomson J (1980) The C. elegans male: postembryonic development of nongonadal structures. Dev Biol 78:542 576.

Thomas JH (1990) Genetic analysis of defecation in Caenorhabditis elegans. Genetics 124:855-872.

Trent $\mathrm{C}$, Tsung N, Horvitz H (1983) Egg-laying defective mutants of the nematode $C$. elegans. Genetics 104:619-647.

Vallés AM, White K (1986) Development of serotonin-containing neurons in Drosophila mutants unable to synthesize scrotonin. J Neurosci 6:1482-1491.

Wang BB, Müller-Immergluck MM, Austin J, Robinson NT, Chisholm A, Kenyon C (1993) A homeotic gene cluster patterns the anteroposterior body axis of $C$. elegans. Cell 74:29-42.

Waring DA, Kenyon C (1990) Selective silencing of cell communication influences anteroposterior pattern formation in C. elegans. Cell 60:123-131.

Waring DA, Wrischnik L, Kenyon C (1992) Cell signals allow the expression of a pre-existent neural pattern in C. elegans. Development 116:457-466.

White J (1988) The anatomy. In: The nematode Caenorhabditis elegans (Wood WB, ed), pp 81-121. Cold Spring Harbor, NY: Cold Spring Harbor Laboratory.

Williams BD, Schrank B, Huynh C, Shownkcen R, Watcrston RH (1992) A genetic mapping system in Caenorhabditis elegans based on polymorphic sequence-tagged sites. Genetics 131:609-624.

Zoran MJ, Haydon PG, Matthews PJ (1989) Aminergic and peptidergic modulation of motor function at an identified neuromuscular junction in Helisoma. J Exp Biol 142:225-243. 